\title{
Application and Use of Multivariate Control Charts In a BTA Deep Hole Drilling Process
}

\author{
Amor Messaoud, Winfied Theis, Claus Weihs, and Franz Hering \\ Fachbereich Statistik, \\ Universität Dortmund, 44221 Dortmund, Germany
}

\begin{abstract}
Deep hole drilling methods are used for producing holes with a high length-to-diameter ratio, good surface finish and straightness. The process is subject to dynamic disturbances usually classified as either chatter vibration or spiralling. In this paper, we will focus on the application and use of multivariate control charts to monitor the process in order to detect chatter vibrations. The results showed that chatter is detected and some alarm signals occurs at time points which can be connected to physical changes of the process.
\end{abstract}

\section{Introduction}

Deep hole drilling methods are used for producing holes with a high length-todiameter ratio, good surface finish and straightness. For drilling holes with a diameter of $20 \mathrm{~mm}$ and above, the BTA (Boring and Trepanning Association) deep hole machining principle is usually employed. The process is subject to dynamic disturbances usually classified as either chatter vibration or spiralling. Chatter leads to excessive wear of the cutting edges of the tool and may also damage the boring walls. Spiralling damages the workpiece severely. The defect of form and surface quality constitute a significant impairment of the workpiece. As the deep hole drilling process is often used during the last production phases of expensive workpieces, process reliability is of primary importance and hence disturbances should be avoided. For this reason, process monitoring is necessary to detect dynamic disturbances.

In this work, we will focus on chatter which is dominated by single frequencies, mostly related to the rotational eigenfrequencies of the boring bar. Therefore, we propose to monitor the amplitude of the relevant frequencies in order to detect chatter vibration as early as possible. In practice, it is necessary to monitor several relevant frequencies because the process is subject to different kind of chatter (i. e., chatter at the beginning of the drilling process, high and low frequency chatter). The first idea is to monitor each relevant frequency separately, using a proposed univariate control chart. This strategy is discussed in section 2. Another solution is to use a multivariate control chart to monitor several relevant frequencies simultaneously, which is investigated in section 3 . In section 4 , the different control charts are applied to real data. 


\section{Monitoring the process using multiple Residual Shewhart control charts}

Weinert et al. (2002) proposed the following general phenomenological model capable of describing the transition from stable operation to chatter in one frequency

$$
\frac{d^{2} M(t)}{d t^{2}}+h(t)\left(b^{2}-M(t)^{2}\right) \frac{d M(t)}{d t}+w^{2} M(t)=W(t),
$$

where $M(t)$ is the drilling torque and $W(t)$ is a white noise process. Theis (2004) described the main features of the variation of the amplitudes of the relevant frequencies, using a logistic function. He showed that his approximation is directly connected to the proposed phenomenological model. In fact, he considered $M(t)$ as a harmonic process

$$
M(t)=R(t) \cos (2 \pi f+\phi)
$$

and showed that

$$
2 \frac{d R(t)}{d t}+h(t) R(t)\left(b^{2}-\frac{R(t)^{2}}{2}\right)=\frac{W(t)}{w}
$$

is the amplitude-equation for the differential equation in (1) if there is only one frequency present in the process. He proved that if his proposed logistic function is the right form for $R(t)$, there is a function $h(t)$ so that equation (2) has a solution. From equation (2), the observed variation in amplitude of the relevant frequencies is described by

$$
R_{t}=\left(1+a_{t}\right) R_{t-1}-a_{t} b_{t} R_{t-1}^{3}+\varepsilon_{t},
$$

where $a_{t}$ and $b_{t}$ are time varying parameters and $\varepsilon_{t}$ is normally distributed with mean 0 and variance $\sigma_{\varepsilon}^{2}$. Messaoud et al. (2004) used the autoregressive part of equation (3) to monitor the variation of the amplitude of the relevant frequencies of the process using residual control charts. They showed that the variation in amplitude of the relevant frequencies of the process can be approximated by the AR(1) model when the process is stable and that the nonlinear term $-a_{t} b_{t} R_{t-1}^{3}$ is not important before chatter.

For the monitoring procedure, the AR(1) model is used to calculate the residuals. The idea behind residual control charts is if the AR(1) model fits the data well, the residuals will be approximately uncorrelated. Then, traditional control charts, such as Shewhart chart can be applied to the residuals. A window of the $m$ recent observations is used to estimate parameters $a, \beta$ and $\sigma_{\epsilon}$ of the linear regression model

$$
R_{t}=\beta+(1+a) R_{t-1}+\epsilon_{t}
$$


and to calculate the residuals, given by

$$
e_{t}=R_{t}-\left(1+\hat{a}_{t-1}\right) R_{t-1}-\hat{\beta}_{t-1}
$$

where $\hat{a}_{t-1}$ and $\hat{\beta}_{t-1}$ are estimates of the regression parameters $a$ and $\beta$ at time $t-1$. The estimated standard deviation of the residuals at time $t-1$ is used to set the control limits of the chart $L C L=-k \sigma_{\epsilon, t-1}$ and $U C L=k \sigma_{\epsilon, t-1}$, where $k$ is constant. The residual Shewhart control chart operates by plotting residuals $e_{t}$ given by equation (4). It signals that the process is out-of-control when $e_{t}$ is outside $U C L$ or $L C L$.

In order to monitor several relevant frequencies, the residual Shewhart is used to monitor the variation in amplitude of each relevant frequency, separately. The resulting monitoring strategy signals an out-of-control condition when any univariate control chart produces an out-of-control signal.

\section{Control charts based on data depth for multivariate processes}

\subsection{Data depth}

Data depth measures how deep (or central) a given point $\mathbf{X} \in R^{d}$ is with respect to (w. r. t.) a probability distribution $F$ or w. r. t. a given data cloud $\left\{\mathbf{Y}_{1}, \ldots, \mathbf{Y}_{m}\right\}$. There are several measurements for the depth of the observations, such as Mahalanobis depth, the simplicial depth, half-space depth, and the majority depth of Singh, see Liu et al. (1999). In this work, the Mahalanobis depth and simplicial depth are considered.

1. The Mahalanobis depth $M D_{F}$ of a given point $\mathbf{X} \in R^{d}$ w. r. t. $F$ is defined to be

$$
M D_{F}(\mathbf{X})=\frac{1}{1+\left(\mathbf{X}-\mu_{F}\right)^{\prime} \Sigma_{F}^{-1}\left(\mathbf{X}-\mu_{F}\right)},
$$

where $\mu_{F}$ and $\Sigma_{F}$ are the mean vector and dispersion matrix of $F$, respectively. The sample version of $M D_{F}$ is obtained by replacing $\mu_{F}$ and $\Sigma_{F}$ with their sample estimates. In fact, how deep $\mathbf{X}$ is w. r. t. $F$ is measured by how small its quadratic distance is to the mean.

2. The simplicial depth $\left(S D_{F}\right)(\mathrm{Liu}, 1990)$ of a given point $\mathbf{X} \in R^{d}$ w. r. t. $F$ is defined to be

$$
S D_{F}(\mathbf{X})=P_{F}\left\{\mathbf{X} \in s\left[\mathbf{Y}_{1}, \ldots, \mathbf{Y}_{d+1}\right]\right\}
$$

where $s\left[\mathbf{Y}_{1}, \ldots, \mathbf{Y}_{d+1}\right]$ is a $d$-dimensional simplex whose vertices are the random observations $\left\{\mathbf{Y}_{1}, \ldots, \mathbf{Y}_{d+1}\right\}$ from $F$. The sample simplicial depth 
$S D_{F_{m}}(\mathbf{X})$ is defined to be

$$
S D_{F_{m}}=\left(\begin{array}{c}
m \\
d+1
\end{array}\right)^{-1} \sum_{1 \leq i_{1}<\cdots<i_{d+1} \leq m} I\left(\mathbf{X} \in s\left[\mathbf{Y}_{1}, \ldots, \mathbf{Y}_{d+1}\right]\right),
$$

where $\left\{\mathbf{Y}_{1}, \ldots, \mathbf{Y}_{m}\right\}$ is a random sample from $F, F_{m}$ denotes the empirical distribution of $\left\{\mathbf{Y}_{1}, \ldots, \mathbf{Y}_{m}\right\}$ and $I($.$) is the indicator function. For$ example, the bivariate $S D_{F_{m}}(\mathbf{X})$ relative to $\left\{\mathbf{Y}_{1}, \ldots, \mathbf{Y}_{m}\right\}$ is equal to the proportion of closed triangles with vertices $\mathbf{Y}_{i}, \mathbf{Y}_{j}, \mathbf{Y}_{k}$ that contain $\mathbf{X}$, $1 \leq i<j<k \leq m$.

\section{$3.2 \quad$ r-chart (Liu, 1995)}

Liu (1995) used the concept of data depth to construct a nonparametric control chart for monitoring processes of multivariate quality measurements, i.e., $k(k \geq 1)$ characteristics are used to determine the quality of the product. The author assumed that the process is considered to be in control if the measurements are following a prescribed distribution, denoted by $F$ (required by customers or designing engineers, ...). If an assignable cause occurs, then any resulting change in the process will be reflected by a location change and/or a scale increase and characterized as a departure from $F$ to a continuous out-of-control distribution $G$.

To design the control chart, a reference sample $\left\{\mathbf{Y}_{1}, \ldots, \mathbf{Y}_{m}\right\}$ of $m$ random observations from $F$ is considered as the measurements of products produced by an in-control process. After the reference sample is obtained, new observations $\mathbf{X}_{1}, \mathbf{X}_{2}, \ldots$ are taken from the process at specified sampling points $t=1,2, \ldots$, respectively. In order to determine if the process is still in-control at time $t$, a statistic $r_{m}\left(\mathbf{X}_{t}\right)$ is calculated

$$
r_{m}\left(\mathbf{X}_{t}\right)=\frac{1}{m} \sum_{i=1}^{m} I\left(D_{m}\left(\mathbf{Y}_{i}\right) \leq D_{m}\left(\mathbf{X}_{t}\right)\right)
$$

where $I($.$) is the indicator function and D_{m}($.$) is the data depth measure. r_{m}$ $\left(0 \leq r_{m} \leq 1\right)$ indicates the fraction of observations in the reference sample $\left\{\mathbf{Y}_{1}, \ldots, \mathbf{Y}_{m}\right\}$ that have data depth measure not exceeding $D_{m}\left(\mathbf{X}_{t}\right)$.

A very small value of $r_{m}$ means that only a very small proportion of $\mathbf{Y}_{i}$ 's is more outlying than $\mathbf{X}_{t}$. Thus $\mathbf{X}_{t}$ is at the "outskirt" of $\left\{\mathbf{Y}_{1}, \ldots, \mathbf{Y}_{m}\right\}$ and is not conforming to most of the central part of the good data set. The main idea behind Liu's (1995) control chart is to "reduce each multivariate measurement to a univariate index-namely, its relative center outward ranking induced by a data depth" measure. The r-chart plots the rank statistic $r_{G_{m}}\left(\mathbf{X}_{t}\right)$ versus time for each observation $\mathbf{X}_{t}$ and compares it to the control limits. It has a center line $C L=0.5$ and a lower control limit $L(0<L<1)$ which is 
equal to the false alarm rate $\alpha$. Note that the r-chart has no upper control limit. In fact, the statistic $r_{G_{m}}$ is "higher the better". The process is said to be out-of-control if $r_{m}<L$. For more details about the motivation and justification of the r-chart see Liu (1995)

\subsection{A control chart based on sequential rank of data depth measures}

Hackl and Ledolter (1992) consider nonparametric control chart procedures for individual observations that use the "standardized ranks" of the observations among the recent group of $m$ observations. In this work, we propose to generalize this control chart to the multivariate case using the notion of data depth. For this chart, the reference sample is considered as the $m>1$ most recent observations taken from the process $\mathbf{X}_{t-m}, \mathbf{X}_{t-m+1}, \ldots, \mathbf{X}_{t-1}$. Therefore, the depth of $\mathbf{X}_{t}$ is calculated w. r. t. this reference sample.

The sequential rank $S_{t}^{*}$ is the rank of $D_{m}\left(\mathbf{X}_{t}\right)$ among $D_{m}\left(\mathbf{X}_{t-m}\right), \ldots$, $D_{m}\left(\mathbf{X}_{t-1}\right)$. That is,

$$
S_{t}^{*}=1+\sum_{i=t-m}^{t-1} I\left(D_{m}\left(\mathbf{X}_{t}\right)>D_{m}\left(\mathbf{X}_{i}\right)\right) .
$$

The standardized sequential rank $S_{t}^{(m)}$ is defined as

$$
S_{t}^{(m)}=\frac{2}{m}\left(S_{t}^{*}-\frac{m+1}{2}\right) .
$$

The control statistic $T_{t}$ is the exponentially weighted moving averages (EWMA) of standardized ranks, computed as follows

$$
T_{t}=(1-\lambda) T_{t-1}+\lambda S_{t}^{(m)},
$$

$t=1,2, \ldots$ and where $T_{0}$ is a starting value, usually set equal to zero, and where $0<\lambda<1$ is a smoothing parameter. The process is considered in-control as long as $T_{t}>h$, where $h<0$ is a lower control limit. In fact, we consider a one sided EWMA chart because $S_{t}^{(m)}$ is "higher the better". For more details, see Hackl and Ledolter (1992).

\section{Application}

The proposed monitoring procedures are used to jointly monitor the amplitudes of frequencies $234 \mathrm{~Hz}$ and $703 \mathrm{~Hz}$, which are among the eigenfrequencies of the boring bar, in an experiment with feed $f=0.185 \mathrm{~mm}$, cutting speed $v_{c}=90 \mathrm{~m} / \mathrm{min}$ and amount of oil $\dot{V}_{\text {oil }}=300 \mathrm{l} / \mathrm{min}$. For more details, see 

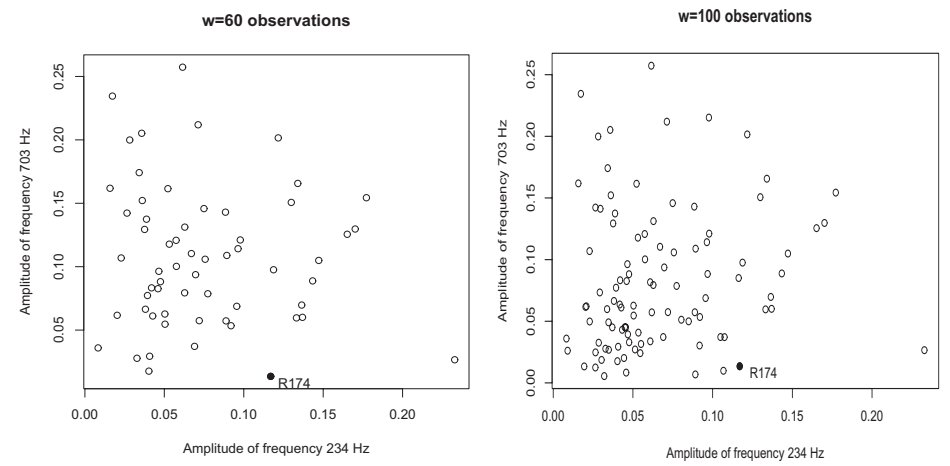

Fig. 1. Effect of reference sample size on the SD measure

Weinert et al. (2002).

For the two residual Shewhart control charts the value 3.09 is chosen for $k$. The $m=100$ observations $R_{t-m, 234}, \ldots, R_{t-1,234}$ and $R_{t-m, 703}, \ldots$, $R_{t-1,703}$ are used to estimate the parameters of the two AR(1) models and to calculate the residuals, see section $2 . R_{t, 234}$ and $R_{t, 703}$ are the actual amplitudes of frequencies $234 \mathrm{~Hz}$ and $703 \mathrm{~Hz}$, respectively. $L=0.01$ is used for the r-chart. For the EWMA chart, after some consideration we used $\lambda=0.3$ and $h=-0.62$. A window of the $m=100$ recent observations $\mathbf{R}_{t-m}, \ldots, \mathbf{R}_{t-1}$, where $\mathbf{R}_{t}=\left(R_{t, 234}, R_{t, 703}\right)^{\prime}$, is used as a reference sample for the r-chart and EWMA chart. The simplicial depth is computed using the FORTRAN algorithm developed by Rousseeuw and Ruts (1992).

Table (1) shows the results, for depth $\leq 270 \mathrm{~mm}$. Table (1) shows that all control charts signal at $32 \leq$ depth $\leq 35 \mathrm{~mm}$. In fact, it is known that approximately at depth $=35 \mathrm{~mm}$ the guiding pads of the BTA tool leave the starting bush, which induce a change in the dynamics of the process. All control charts (except the EWMA using MD) signal at depth $110 \leq$ depth $\leq 120$ $\mathrm{mm}$ and it is known that depth $110 \mathrm{~mm}$ is approximately the position where the tool enters the bore hole completely. Theis (2004) noted that this might lead to changes in the dynamic process because the boring bar is slightly thinner than the tool and therefore the pressures in the hole may change. The important out-of-control signals are produced at $250 \leq$ depth $\leq 255$ $\mathrm{mm}$. Messaoud et al. (2004) showed that a change occurred in the process at depth $=252.19 \mathrm{~mm}$ and they concluded that this change may indicate the presence of chatter or that chatter will start in few seconds.

Table (1) shows that the r-chart using SD produced 99 out of control signals. Indeed, it is known that $\mathrm{r}$-charts require a large reference sample size. Stoumbos and Reynolds (2001) demonstrated that these charts appear 
to have limited potential for practical applications due to this requirement. Figure 1 shows the effect of reference sample size $m$ on the SD measure. Observation $\mathbf{R}_{174}=\left(R_{174,234}, R_{174,703}\right)^{\prime}$ is outside the data cloud using $m=60$ observations. However, when $m=100, \mathbf{R}_{174}$ is further inside the data cloud and is contained in some triangles. The proposed EWMA based on sequential rank of SD measures produced 16 out of control signals, which indicates that this chart can be used to monitor the process.

Table 1. Out of control signals of the different control charts applied to the amplitude of frequencies $234 \mathrm{~Hz}$ and $703 \mathrm{~Hz}(m=100)$

\begin{tabular}{|c|c|c|c|c|c|}
\hline \multirow[t]{2}{*}{$\begin{array}{l}\text { Hole Depth } \\
\text { (mm) }\end{array}$} & \multirow[t]{2}{*}{$\begin{array}{c}\text { Residual } \\
\text { Shewharts }\end{array}$} & \multicolumn{2}{|c|}{$\begin{array}{c}\mathrm{r} \text {-chart } \\
r_{m}<.01\end{array}$} & \multicolumn{2}{|c|}{$\begin{array}{c}\text { EWMA }(\lambda=0.3) \\
T_{t} \leq-.62\end{array}$} \\
\hline & & $\mathrm{MD}$ & SD & $\mathrm{MD}$ & $\mathrm{SD}$ \\
\hline$<32$ & 0 & 0 & 0 & 0 & 0 \\
\hline $32-35$ & 1 & 1 & 3 & 3 & 2 \\
\hline $35-45$ & 4 & 2 & 11 & 13 & 6 \\
\hline $45-70$ & 2 & 1 & 7 & 0 & 0 \\
\hline $70-110$ & 2 & 0 & 17 & 0 & 1 \\
\hline $110-120$ & 4 & 1 & 8 & $\mathbf{0}$ & 1 \\
\hline $120-200$ & 3 & 6 & 29 & 0 & 2 \\
\hline $200-250$ & 3 & 4 & 16 & 0 & 1 \\
\hline $250-255$ & 1 & 1 & 2 & 4 & 2 \\
\hline $255-260$ & 0 & 0 & 2 & 1 & 1 \\
\hline $260-270$ & 0 & 0 & 4 & 0 & 0 \\
\hline Total & 20 & 16 & 99 & 21 & 16 \\
\hline
\end{tabular}

\section{Conclusion}

A main objective of this work is to investigate whether multivariate control charts can be used to monitor the drilling process. The results showed that the different control charts can detect chatter and that some out-of-control signals are related to changing physical conditions of the process (i.e., guiding pads leave the starting bush, the tool is completely in the hole).

Multiple residual Shewhart assume independence and normality of the residuals, see Messaoud et al. (2004), and in practice it is difficult to interpret multiple control charts. Multivariate control charts based on data depth are "distribution-free" control charts and are easy to visualize and interpret.

Based on the practical results, we feel that the proposed EWMA chart using SD can be used to monitor the process. However, further investigation of the statistical properties of the chart are needed. Moreover, the future 
research should focus on the out-of-control interpretation. In fact, when the control chart indicates an out-of-control condition, it is important to determine which frequency, or combination of frequencies, of the multivariate process caused the process to go out-of-control. In practice, the identification of the type of chatter (i.e., chatter at the beginning of the drilling process, low-high frequency chatter) will usually make it easier for engineers to adjust the process.

\section{Acknowledgements}

This work has been supported by the Graduate School of Production Engineering and Logistics at the university of Dortmünd and the Collaborative Research Centre "Reduction of Complexity in Multivariate Data Structures" (SFB 475) of the German Research Foundation (DFG).

\section{References}

HACKL, P. and LEDOLTER, J. (1991): A New Nonparametric Quality Control Technique. Communications in Statistics-Simulation and Computation 21, 423-443.

LIU, Y. R. (1990): On a notion of data depth based on random simplices. The Annals of Statistics, 18, 405-414.

LIU, Y. R. (1995): Control Charts for multivariate Processes. Journal of the American Statistical Association, 90, 1380-138\%.

LIU, Y. R.; PARELIUS, J. M.; and SINGH, K. (1999): Multivariate analysis by data depth: Descriptive Statistics, Graphics and Inference. The Annals of Statistics, 27, 783-858.

MESSAOUD, A.; THEIS, W.; WEIHS, C.; and HERING, F. (2004): Monitoring the BTA Deep Hole Drilling Process Using Residual Control Charts. Unpublished paper, available from the first author.

ROUSSEEUW, P. and RUTS, I. (1996): AS 307: bivariate location depth. Applied Statistics, 45, 516-526.

STOUMBOS, Z. G. and REYNOLDS Jr, M. R. (2001): On Shewhart-Type Nonparametric Multivariate Control Charts Based on Data Depth. Frontiers in Statistical Quality Control 6, 207-22\%.

THEIS, W. (2004): Modelling Varying Amplitudes. PhD dissertation, Department of Statistics, Universität Dortmund.

WEINERT, K.; WEBBER, O.; HÜSKEN, M.; MENHEN, J.; and THEIS, W. (2002): Analysis and prediction of dynamic disturbances of the BTA deep hole drilling process. Proceedings of the $3^{\text {rd }}$ CIRP International Seminar on Intelligent Computation in Manufacturing Engineering. 